\title{
Angioma cavernoso del trígono ventricular. Caso clínico
}

\author{
J.M. González-Darder; J.V. Pesudo-Martínez y J. Merino-Peña
}

Servicio de Neurocirugía. Hospital General de Castellón. Castellón de la Plana.

\section{Resumen}

Se presenta un caso de cavernoma intraventricular de trígono derecho en un hombre de 25 años con sangrado espontáneo predominantemente intralesional. Las técnicas de imagen permitieron el diagnóstico preoperatorio de la lesión, aunque faltaba el anillo perilesional de gliosis y hemosiderina. La lesión fue extirpada microquirúrgicamente sin incidencias por vía temporal posterior trans-sulcal y con guía estereotáctica.

PALABRAS CLAVE: Angioma cavernoso. Trígono ventricular.

Trigonal cavernous angioma: case report

\section{Summary}

The authors report on an intraventricular cavernous angioma located at the right trigone in a 25year-old male patient presented with a predominantly intralesional haemorrhage. Neuroimaging led to an accurate preoperative diagnosis although the typical low intensity perilesional ring of gliosis and hemosiderin was not present. The lesion was microsurgically removed using an stereotactically guided posterior temporal transsulcal approach.

Key words : Cavernous angioma. Ventricular trigone.

El angioma cavernoso o cavernoma intraventricular es una lesión poco frecuente ${ }^{3,5,6}$ y que tiene unas características clínicas y de imagen diferentes a las de los cavernomas intraparenquimatosos ${ }^{3-5,7-9}$. En este trabajo se comunica un caso de cavernoma intraventricular localizado en el trígono, revisando sus características clínicas y diagnósticas.

Recibido: 9-10-06. Aceptado: 13-10-06

\section{Caso clínico}

Hombre de 25 años, sin antecedentes de interés, diestro, que debuta un año antes de la cirugía con un cuadro de cefalea brusca, desorientación y visión borrosa. Una tomografía axial computarizada (TAC) craneal mostraba una imagen intraventricular a nivel de trígono derecho sugestiva de sangrado, con muy discreto realce irregular tras la inyección de contraste (fig.1A). La evolución fue favorable, quedando libre de síntomas y se remitió meses mas tarde para valoración neuroquirúrgica. Se practicó una resonancia nuclear magnética (RNM) cerebral que mostraba una lesión de 2cm, irregular, con sangre en diferentes estadios evolutivos y con captación mínima y muy irregular de contraste (fig.1B-E). Se realizó una angiografía cerebral que no demostraba vascularización patológica. Con el diagnóstico preoperatorio de cavernoma se indicó la cirugía. El paciente fue intervenido bajo anestesia general a través de un abordaje trans-sulcal temporal posterior guiado estereotácticamente. Se identificó una lesión con las características típicas del cavernoma, intraventricular y creciendo en el plexo coroideo, que fue extirpada en bloque sin incidencias con técnica microquirúrgica (Fig.1F). El estudio anatomopatológico confirmó el diagnóstico. El paciente evolucionó favorablemente y fue dado de alta sin secuelas a los cuatro días de la cirugía.

\section{Discusión}

El cavernoma o angioma cavernoso de localización intraventricular es una lesión poco descrita en la literatura. Reyns et al. recogían 45 casos identificados después de la introducción del TAC como medio de diagnóstico ${ }^{5}$. Más recientemente, una revisión del tema realizada por Suess et al. reúne un total de 50 casos publicados ${ }^{8}$. La frecuencia de cavernomas intraventriculares es de menos del $10 \%$ de los cavernomas intracraneales La localización mas frecuente del cavernoma intraventricular es el tercer ventrículo y los ventrículos laterales, mientras que la localización en el trígono es mucho mas rara ${ }^{3,5}$.

Kumar et $\mathrm{al}^{3}$ encuentran en la literatura un total de 9 


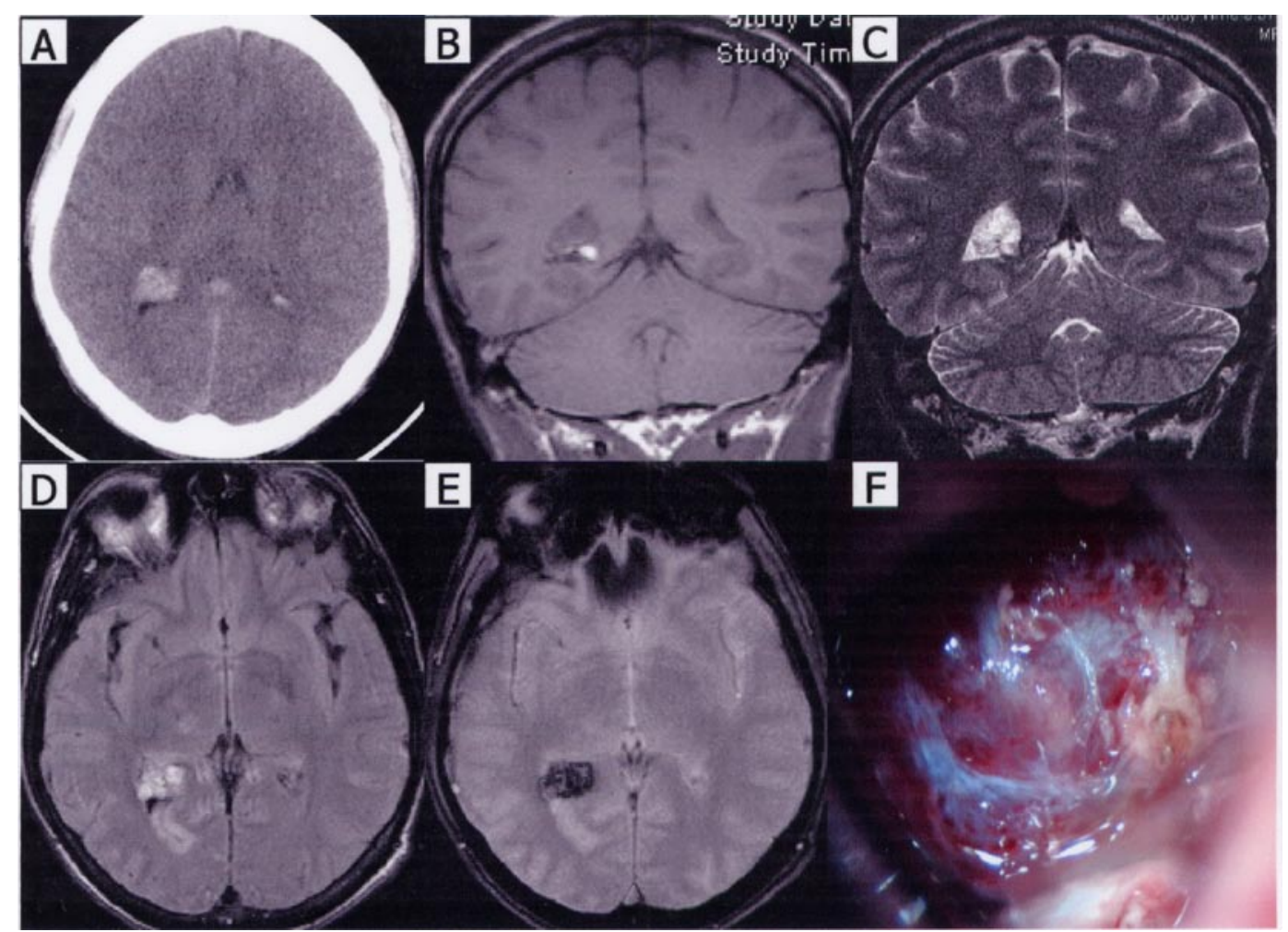

Figura 1. TAC sin contraste (A), mostrando una lesión en el trígono derecho, hiperintensa e irregular. Imágenes de RNM en secciones coronales potenciadas en $T 1$ (B) y T2 (C) y secciones axiales en flair (D) y gradienteeco T2 (E), donde se aprecia un cambio de señal en el asta occipital. Fotografia intraoperatoria, con el aspecto característico del cavernoma intraventricular $(F)$.

casos de cavernoma del trígono ventricular, a los que hay que añadir el publicado por Tatsui et $\mathrm{al}^{9}$, el incluido en la serie de tumores intraventriculares de Gaab y Schroeder ${ }^{2}$ y el descrito en este trabajo. La presentación es más frecuente en pacientes jóvenes, con la tercera parte de ellos en edad pediátrica. La manifestación clínica mas frecuente es la hipertensión endocraneal y epilepsia, lo que se debería a que el tamaño de la lesión en el momento del diagnóstico es grande $(3-5 \mathrm{~cm})$ al poder crecer libremente en la cavidad intraventricular. Sólo un caso, además del nuestro, se presentó con hemorragia intraventricular. Esta escasa presentación hemorrágica contrasta con la de los cavernomas intraparenquimatosos, donde la clínica de presentación hemorrágica varía entre el 30 y 50\% según las series ${ }^{7}$. Además, en nuestro paciente la hemorragia debe considerarse eminentemente intralesional, ya que no hubo clínica ni imagen de hemorragia intraventricular, aunque en la RNM tardía se aprecian cambios periventriculares en el fondo del asta occipital sugestivos de cierto grado de trasudado hemático. El sangrado intralesional es una complicación evolutiva, es una forma de presentación sintomática frecuente y se considera como una de las formas de crecimiento del cavernoma. Los cavernomas del tercer y cuarto ventrículo se presentan también clínicamente a través de su efecto de masa y por hidrocefalia, mientras que en los ventrículos laterales la incidencia de epilepsia y sangrado es mayor ${ }^{6}$.

Los hallazgos de imagen son los habituales para el cavernoma intraparenquimatoso $0^{3-9}$. Sin embargo, puede no haber el típico anillo de hiposeñal en las series potenciadas en T2 o de eco-gradiente tan característico del cavernoma cerebral. Esto sería debido al hecho de que las pequeñas hemorragias periféricas o el trasudado de los restos hemáticos se lavaría al caer en el líquido cefalorraquídeo, impidiendo el acumulo de hemosiderina y el desarrollo de la gliosis reactiva del tejido nervioso. En nuestro caso y en otros descritos no se encuentra este signo ${ }^{4,9}$.

El diagnóstico diferencial del cavernoma intraventricular de trígono es más limitado que en el caso de los de localización en el tercer ventrículo o ventrículos laterales. Los tumores intraventriculares de trígono mas frecuentes son el papiloma de plexos coroideo, gliomas y el meningioma, pero ante una lesión que ha sangrado debe considerarse una malformación arteriovenosa que se excluye con el estudio angiográfico.

El tratamiento del cavernoma intraventricular trígono sintomático es quirúrgico. La vía de abordaje dependerá del tamaño y dominancia del hemisferio donde se encuentre $^{6}$. En este sentido, puede ser interesante en el estudio preoperatorio la realización de una RNM-funcional con paradigmas que estudien la función verbal ${ }^{1}$. Dado que no es frecuente la hidrocefalia asociada y que el tamaño de la lesión suele ser grande, sólo excepcionalmente se puede realizar una resección por vía endoscópica². Por esta misma razón, es aconsejable usar una técnica de guiado estereotático o de neuronavegación, con abordaje microquirúrgico 
trans-sulcal. El aspecto quirúrgico de la lesión es patognomónico y se encuentra creciendo en el plexo coroideo, aunque lesiones grandes pueden adherirse al epéndimo, mientras que en casos de auténtico crecimiento desde el parénquima, podrían tener un origen subependimario con posterior ocupación del espacio intraventricular ${ }^{3,4}$. El pronóstico tras la resección completa es bueno, por lo que ante el diagnóstico diferencial de una masa localizada en el trígono ventricular debe considerarse la posibilidad de un cavernoma para evitar maniobras diagnósticas eventualmente peligrosas como la biopsia esterotáctica o actitudes ineficaces como la observación o la radiocirugía ${ }^{5}$.

\section{Bibliografía}

1. Avila, C., Barros-Loscertales, A., Parcet-Ibars, M.A., Belloch-Ugarte, V., Campos-Hernández, S., Feliu-Tatay, R., González-Darder, J.M.: Aplicaciones de la resonancia nuclear magnética funcional en pacientes prequirúrgicos: funciones motora, de memoria y lingüística. Rev Neurol 2003; 37: 567578.

2. Gaab, M.R., Schroeder, H.W.: Neuroendoscopic approach to intraventricular lesions. J Neurosurg 1998; 88: 496505.

3. Kumar, G.S.S., Poonnoose, S.I., Chaco, A.G., Rajshekhar, V.: Trigonal cavernous angiomas: report of three cases and review of literature. Surg Neurol 2006; 65: 367-371.
4. Nieto, J., Hinojosa, J., Muñoz, M.J., Esparza, J., Ricoy, J.R.: Intraventricular cavernoma in pediatric age. Childs Nerv Syst 2003; 19: 60-62.

5. Suess, O., Hammarse, S., Brock, M.: Intraventricular cavernoma: unusual occurrence in the region of the foramen of Monro. Br J Neurosurg 2002 ; 16: 78-79.

6. Reyns, N., Assaker, R., Louis, E., Lejeune, J.P.: Intraventricular cavernomas: three cases and review of the literature. Neurosurgery 1999; 44: 648-654.

7. Rhoton, A.L.: The lateral and third ventricles. Neurosurgery 2002; 51 (suppl 1): 207-271.

8. Rigamonti, D., Hsu, F.P.K., Huhn, S.: Angiographically occult vascular malformations. En: Carter LP, Spetzler RF. Neurovascular Surgery, McGraw-Hill, New York, 1995, pp 521-540.

9. Tatsui, C.E., Koerbel, A., Prevedello, D.M.S., Hanel, R.A., Grande, C.V., Moro, M.S., Araujo, J.C.: Ressonáncia magnética dos cavernomas intraventriculares. Aspectos diagnósticos. Arq Neuropsichiatr 2003; 61: 79-82.

González-Darder, J.M.; Pesudo-Martínez, J.V.; MerinoPeña, J.: Angioma cavernoso del trígono ventricular. Caso clínico. Neurocirugía 2007; 18: 330-332.

Correspondencia postal: José M González-Darder. Servicio de Neurocirugía. Hospital General de Castellón. Avda. Benicassim s/n 12004 Castellón. 